\section{BENCH TO BeDside}

\section{Deadly 1918 flu remade}

The I9I8 influenza pandemic was unusually devastating, killing an estimated $20-50$ million people worldwide. In the years since, no influenza $A$ virus has struck healthy young adults in the same way, leaving the virulence of the I9I8 flu as a historical oddity. Now, there is evidence that the virus responsible for the 1918 pandemic is related to the current avian virus that has scientists fearing a similar worldwide outbreak.

Terrence Tumpey and colleagues recently resurrected the I9I8 influenza virus (Science 2005;310:77-80). They did so by reconstructing its genes from lung materials archived from autopsies and tissues preserved from an Alaskan influenza victim who was buried in the permafrost.

Performing experiments under strict biosafety precautions and while taking antiviral prophylactic drugs, the scientists reconfirmed the lethality of infection with the 1918 virus in mice. Those infected with the virus died after 3-5 days, whereas those who received contemporary strains of influenza had transient weight loss only.

Modern molecular biology is also uncovering reasons for the virulence of the 1918 virus. Tumpey and colleagues found that the IgI8 virus can replicate in the absence of the host protein trypsin, which is normally required by influenza viruses; the IgI8 strain therefore may be able to grow in a variety of cell types beyond simply trypsincovered lung cells. The authors then confirmed the lethality of the I9I8 virus in chicken embryos. This finding is important: although current human influenza viruses do not have this effect, the avian virus certainly does.

In a complementary paper, Jeffery Taubenberger and coworkers have revealed that the reconstructed 1918 virus is indeed more related to avian flu viruses than to human flu viruses ( $\mathrm{Na}$ ture 2005;437:889-93). This, they note,

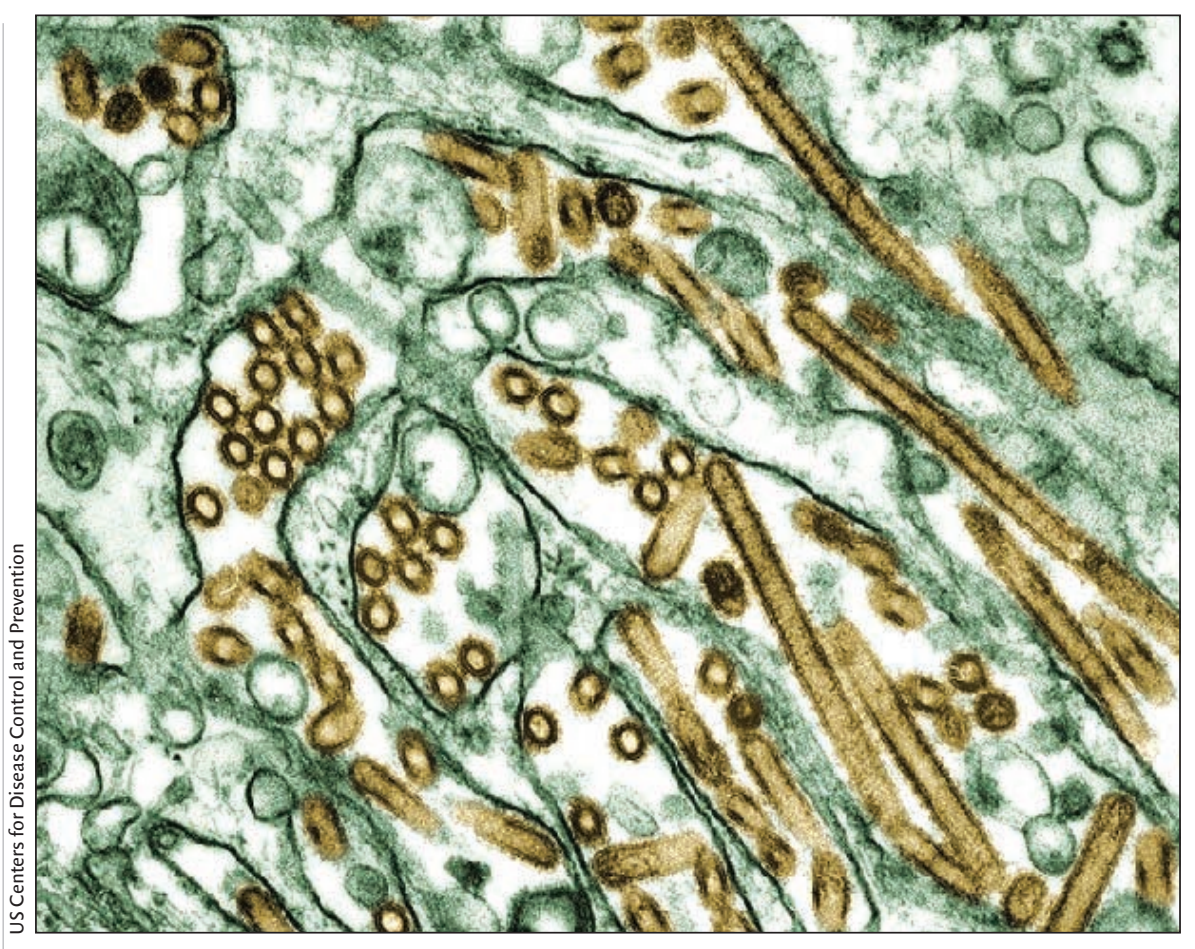

Electron micrograph of avian influenza virus (seen in gold).

suggests that the I9I 8 virus evolved from an avian source.

Amid growing concern that the avian virus will evolve to cause a future pandemic, an understanding of the related 1918 virus may lead to treatments that might permit us to avoid a global catastrophe.

\section{Avian flu shows resistance}

The threat of a global flu pandemic caused by the $\mathrm{H}_{5} \mathrm{NI}$ avian influenza virus has many countries racing to stockpile the antiviral drug oseltamivir, which targets the viral enzyme neuraminidase and inhibits the release of viral progeny from a cell. The drug is well tolerated and effective against a variety of influenza strains. Together with zanamivir (a neuraminidase inhibitor), oseltamivir is also considered a good prophylactic, since it appears not as prone to the development of viral resistance as other drugs ( $N$ Engl $J$ Med 2005;353:1363-73).

On the other hand, a recent report that $\mathrm{H}_{5} \mathrm{NI}$ can be resistant to oseltam- ivir has suggested that this drug alone may not be enough to cope. A case of avian influenza in Vietnam in which the virus was resistant to oseltamivir was reported by Q. Mai Le and associates (Nature 2005; doi:10.1038/4371108a). The affected girl had had no contact with poultry, but had cared for her $\mathrm{H}_{5} \mathrm{NI}$-infected brother. The exposure raises concern of human-to-human transmission of the virus, an essential step in the ability of the virus to cause an epidemic.

Le and associates went on to establish that the oseltamivir-resistant $\mathrm{H}_{5} \mathrm{NI}$ strain has a single amino-acid change only (histidine to tyrosine) in its neuraminidase protein. However, when injected into ferrets, the oseltamivirresistant strain was still sensitive to zanamivir. This, the authors suggest, may point to the need for zanamivir stockpiles in addition to oseltamivir, as well as further monitoring for drug resistance. - Compiled by David Secko, Vancouver

DOI:I0.1503/cmaj.051372 